\title{
Impact of an online writing aid tool for writing a randomized trial report: the COBWEB (Consort-based WEB tool) randomized controlled trial
}

Caroline Barnes ${ }^{2,3}$, Isabelle Boutron ${ }^{1,2,3^{*}}$, Bruno Giraudeau ${ }^{3,4}$, Raphael Porcher ${ }^{1,2,3}$, Douglas G Altman ${ }^{5}$ and Philippe Ravaud ${ }^{1,2,3,6}$

\begin{abstract}
Background: Incomplete reporting is a frequent waste in research. Our aim was to evaluate the impact of a writing aid tool (WAT) based on the CONSORT statement and its extension for non-pharmacologic treatments on the completeness of reporting of randomized controlled trials (RCTs).

Methods: We performed a 'split-manuscript' RCT with blinded outcome assessment. Participants were masters and doctoral students in public health. They were asked to write, over a 4-hour period, the methods section of a manuscript based on a real RCT protocol, with a different protocol provided to each participant. Methods sections were divided into six different domains: 'trial design', 'randomization', 'blinding', 'participants', 'interventions', and 'outcomes'. Participants had to draft all six domains with access to the WAT for a random three of six domains. The random sequence was computer-generated and concealed. For each domain, the WAT comprised reminders of the corresponding CONSORT item(s), bullet points detailing all the key elements to be reported, and examples of good reporting. The control intervention consisted of no reminders. The primary outcome was the mean global score for completeness of reporting (scale 0-10) for all domains written with or without the WAT.
\end{abstract}

Results: Forty-one participants wrote 41 different manuscripts of RCT methods sections, corresponding to 246 domains (six for each of the 41 protocols). All domains were analyzed. For the primary outcome, the mean (SD) global score for completeness of reporting was higher with than without use of the WAT: 7.1 (1.2) versus 5.0 (1.6), with a mean $(95 \% \mathrm{Cl})$ difference $2.1(1.5-2.7 ; P<0.01)$. Completeness of reporting was significantly higher with the WAT for all domains except for blinding and outcomes.

Conclusion: Use of the WAT could improve the completeness of manuscripts reporting the results of RCTs.

Trial registration: Clinicaltrials.gov (http://clinicaltrials.gov NCT02127567, registration date first received April 29, 2014) Keywords: Clinical epidemiology, CONSORT statement, Randomized controlled trial, Reporting guidelines, Transparency

\footnotetext{
*Correspondence: isabelle.boutron@aphp.fr

${ }^{1}$ Centre d'Épidémiologie Clinique, Hôpital Hôtel Dieu, Assistance Publique

des Hôpitaux de Paris, Hôpital Hôtel Dieu, Aile A2 1er étage 1, Place du

parvis Notre Dame, 75181 Paris, Cedex 4, France

${ }^{2}$ Paris Descartes University, Paris, France

Full list of author information is available at the end of the article
} 


\section{Background}

Inadequate reporting is a frequent cause of waste in research [1, 2]. To overcome this issue, the CONSORT Statement [3], an evidence-based, minimum set of recommendations for reporting randomized controlled trials (RCTs), was developed, along with extensions for reporting specific designs (e.g. cluster [4], non-inferiority [5]), data (e.g. harms [6]), and interventions (nonpharmacologic treatments [7]). The CONSORT guidelines have received support from the World Association of Medical Editors, the Council of Science Editors, and the International Committee of Medical Journal Editors. According to the CONSORT website [8], currently 585 journals worldwide endorse CONSORT. Most journals include recommendations to follow the main CONSORT statement in their instructions to authors and some have specific policies to enforce the use of the CONSORT guidelines at the submission or revision stage of a manuscript [9].

However, the adherence of authors to these guidelines is low and the quality of reporting remains insufficient [9-21]. For example, the method of randomization was described in only $25 \%$ of published RCTs [10, 15, 22], and more than $30 \%$ of reports did not provide sufficient details to allow replication of the treatment evaluated in the trial in clinical practice $[11,23]$.

Several barriers can explain the lack of adherence to the CONSORT guidelines [1, 24-27]. Our hypothesis is that the strategy currently used to implement the reporting guidelines, such as the CONSORT Statement and its extensions, could be improved. First, the timing of implementation of CONSORT is late in the process. In fact, most active strategies used to improve the quality of reporting are implemented by editors and consist of asking authors to submit the CONSORT checklist at the submission or acceptance stage [9, 20]. Modifying a manuscript ready for submission is probably more difficult than writing the first draft of the manuscript according to the guidelines. Implementation at an earlier stage when writing the manuscript, instead of the submission or revision stages, could be more efficient.

Second, the CONSORT Statement and its extension are disseminated in two formats: (1) the CONSORT Statement (a checklist and flow diagram) and (2) the CONSORT "Explanation and Elaboration" document $[3,7]$. The checklist is probably not sufficient for some authors to adequately understand what should be reported. For example, to report the item dedicated to the intervention "Description of the different components of the interventions [..]" authors reporting a surgical trial may benefit from more support to understand that they should report, in addition to the surgical procedure, all of the following information: details on preoperative care, postoperative care, and anesthesia management.
The Explanation and Elaboration documents are meant to help authors understand the checklist. However, these documents are very long (more than 30 pages) and they combine explanations about why the item should be reported, how the item is reported in the literature, what should be reported, and examples of adequate reporting. Consequently, the important information is buried in the manuscript. The use of a template shell with a clear and explicit reminder of what should be reported when authors are writing their manuscript could be useful to increase adherence to the guidelines.

Finally, authors may need more support to adequately combine the different extensions of the CONSORT statement. In fact, to adequately report a cluster RCT evaluating a rehabilitation program, authors must rely on three checklists and three Elaboration and Explanation documents for the main CONSORT, the extension for cluster trials, and the extension for non-pharmacologic treatments. Up to now, no support has been developed to help authors successfully adhere to reporting guidelines at the writing stage of the manuscript.

An online writing aid tool that combines the different extensions of the CONSORT Statement and provides appropriate explanations with examples of adequate reporting would be a more efficient way to implement the CONSORT statements and thus improve the quality of reporting. We developed a writing aid tool based on the CONSORT guidelines and its extension for nonpharmacologic treatments to help authors when writing a report of a RCT. We evaluated the impact of this tool on the completeness of reporting of two-arm parallel-group RCTs evaluating pharmacologic and non-pharmacologic interventions.

\section{Methods}

\section{Trial design}

We performed a 'split-manuscript' RCT with blinded outcome assessment. The methods section of each manuscript was divided into six different domains: 'trial design', 'randomization,' 'blinding,' 'participants,' 'interventions', and 'outcomes'. Each participant was randomly allocated to a different real RCT protocol. Participants had to write the six domains of the methods section of the manuscript for the protocol they received over a 4-hour period. They had access to the tool for a random three of the six domains. Thus, the unit of randomization was the domain, embedded within the manuscript (Fig. 1).

Authorization by the CNIL ("Commission Nationale de l'Informatique et des Libertés"; file number 1753007) whose remit is to protect participants' personal data and the institutional review board of INSERM ethics committee (IRB 00003888) was obtained and the study protocol was registered at ClinicalTrials.gov (http://clinicaltrials.gov NCT02127567). 


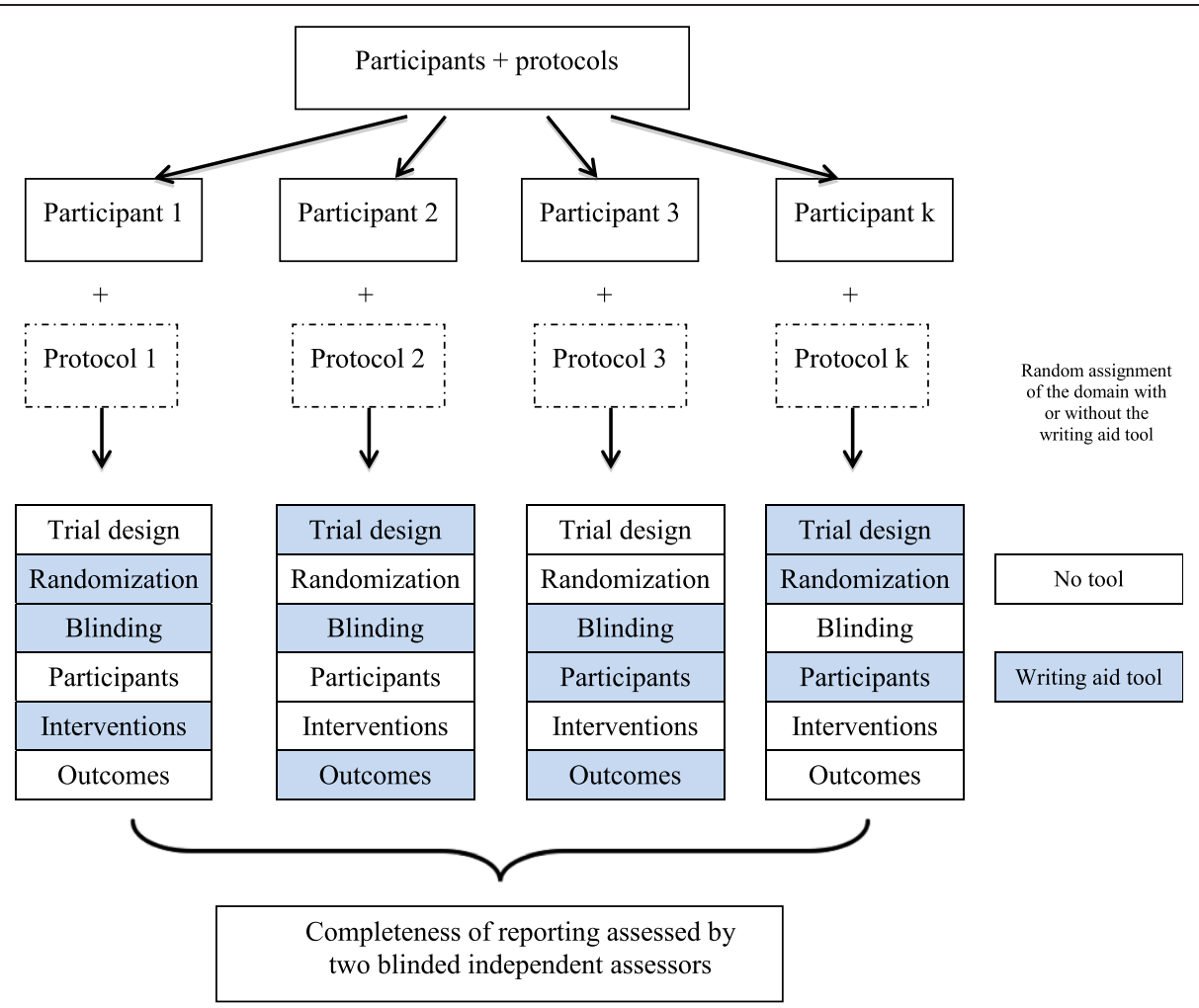

Fig. 1 Schema of the study design

Informed consent was obtained from all participants. The consent was obtained electronically. All parts of the trial were conducted in Paris.

\section{Randomization}

\section{Sequence generation}

The randomization sequence was computer-generated with the use of SAS 9.2. For each participant-manuscript, three domains were allocated to the 'writing aid tool' group and the three remaining domains were allocated to the 'usual writing' group. Allowing for 20 possible combinations of domains (i.e. three of six domains with the tool and three without), randomization was performed with permuted blocks of 20 .

\section{Implementation}

Only the independent statistician and the computer programmer who developed the online writing aid tool and the website had access to the randomization list. The statistician who generated the list (BG) provided the list to the programmer, who uploaded it on the study's secure website. The list was not available to the researchers who enrolled the participants and were present at the various study sessions $(\mathrm{CB}, \mathrm{IB})$.

\section{Allocation concealment}

The sequence was concealed by a computer interface.

\section{Blinding}

Participants could not be blinded to intervention assignments. However, outcome assessors were blinded to intervention assignments.

\section{Participants}

Study participants were masters or doctoral students in the field of public health and medical research who were based in Paris and who were affiliated with Paris Descartes University, Pierre and Marie Curie University, and Paris Diderot University, or the Mailman School of Public Health of Columbia University, in New York.

An e-mail advertisement was sent to students to invite them to participate in a writing session. Participants were not informed of the study in the email advertisement. Before obtaining their consent, participants attended a small informational session with a PowerPoint presentation describing the writing task to perform. Participants were instructed to complete six sections of a manuscript describing the study protocol they were provided and that they would have assistance for three sections and no assistance for three sections, although they were not instructed as to which sections. They were informed that this design had a pedagogical purpose as they could see how useful it was to have the writing aid tool and use reporting guidelines when writing the first draft of a manuscript. They were instructed that we would use their 
results to evaluate the impact of the tool. Before beginning the exercise, participants provided their consent electronically.

\section{Selection of protocols}

We retrieved all protocols of RCTs published between January 1, 2013, and March 28, 2014, in the New England Journal of Medicine or the Journal of Clinical Oncology. We chose these journals because they provide access to the protocol for all the RCTs they publish.

One researcher (CB) searched MEDLINE via PubMed (search strategy is reported in Additional file 1) and screened all titles and abstracts retrieved to select all reports of two-arm parallel-group RCTs. All available protocols published in English were retrieved for all identified reports of RCTs. Then, we constituted a sample of protocols reporting various pharmacologic interventions and non-pharmacologic treatments (surgery, implantable devices, rehabilitation, education, etc.; see sample size below).

\section{Experimental interventions Objective of the tool}

The writing aid tool based on CONSORT was developed to provide guidance to authors when writing a manuscript of a RCT evaluating pharmacologic treatment or non-pharmacologic treatment. The tool was individualized according to the type of treatment evaluated (drug, surgery, participative interventions such as rehabilitation, education).

\section{Content of the tool}

The content of the tool was based on the checklist and the Explanation and Elaboration document for CONSORT 2010 [3] and the checklists and the Explanation and Elaboration documents of the CONSORT extension for nonpharmacologic treatment [7]. For each domain, the tool comprised the corresponding CONSORT checklist item(s), bullet points with the key elements that need to be reported extracted from the Explanation and Elaboration document of the CONSORT 2010, and non-pharmacologic treatment extension, as well as (an) example(s) of good reporting. For the domain dedicated to the intervention, the bullet points and examples of adequate reporting were individualized according to the treatment evaluated (i.e. medication or treatment strategy; surgical procedures or devices; or participative interventions such as rehabilitation, education, behavioral treatment, or psychotherapy). For example, when the experimental treatment was a surgical procedure, the bullet points with the key elements that needed to be reported were specific to surgical procedures (e.g. anesthesia, preoperative care, postoperative care) and the examples of adequate reporting concerned surgical trials.
An example is included in Fig. 2. The entire tool is available at [28] (enter any username) and in Additional file 2.

\section{Format of the tool}

For each domain, the online writing aid tool consisted of a single or several large text boxes in which the participants could write the corresponding part of the methods section. Above each text box was a reminder of the information that should be reported. This reminder consisted of the related CONSORT item followed by the statement "Please describe" and bullet points with all the information that needed to be reported.

According to the domain and the complexity of the CONSORT item, the tool could contain one or several text boxes. For example, two boxes were dedicated to the domain trial design: one to describe the trial design and one to report important changes to methods after the trial commencements with reasons.

\section{Control intervention}

For each domain, the intervention consisted of a large text box in which the participant could write this part of the methods section. They did not have the CONSORT checklist item.

\section{Writing session}

Interventions were administered in the context of a practice writing session. Participants were asked to write six sections of a manuscript based on a protocol they were provided describing an RCT over a 4-hour period. Each participant was provided a protocol randomly selected in our sample of protocol in both electronic copy and paper copies. Two study monitors (CB and IB) supervised these writing sessions after providing a brief explanation of the task to be performed. Participants were aware that they would have access to the writing aid tool for some of the sections. They were not allowed to use any materials. Participants were told that all data would be anonymous and confidential.

For both the experimental and control interventions, participants were instructed to indicate any important or necessary information they would have wished to report that was not available in the provided study protocol (Fig. 2).

\section{Outcomes}

\section{Primary outcome}

The primary outcome was the mean global score for completeness of reporting (scale 0-10) for all domains written with or without the writing aid tool.

For each domain, within each protocol selected, we prespecified a series of keywords that should be reported. For example, in a study using a 1:1 randomization with a computer generated randomization list with blocks of four and 
The writing tool prompted the writer to describe both the experimental and then control intervention separately. Below is an example of the writing aid for describing the experimental intervention for non pharmacological behavioral based interventions.

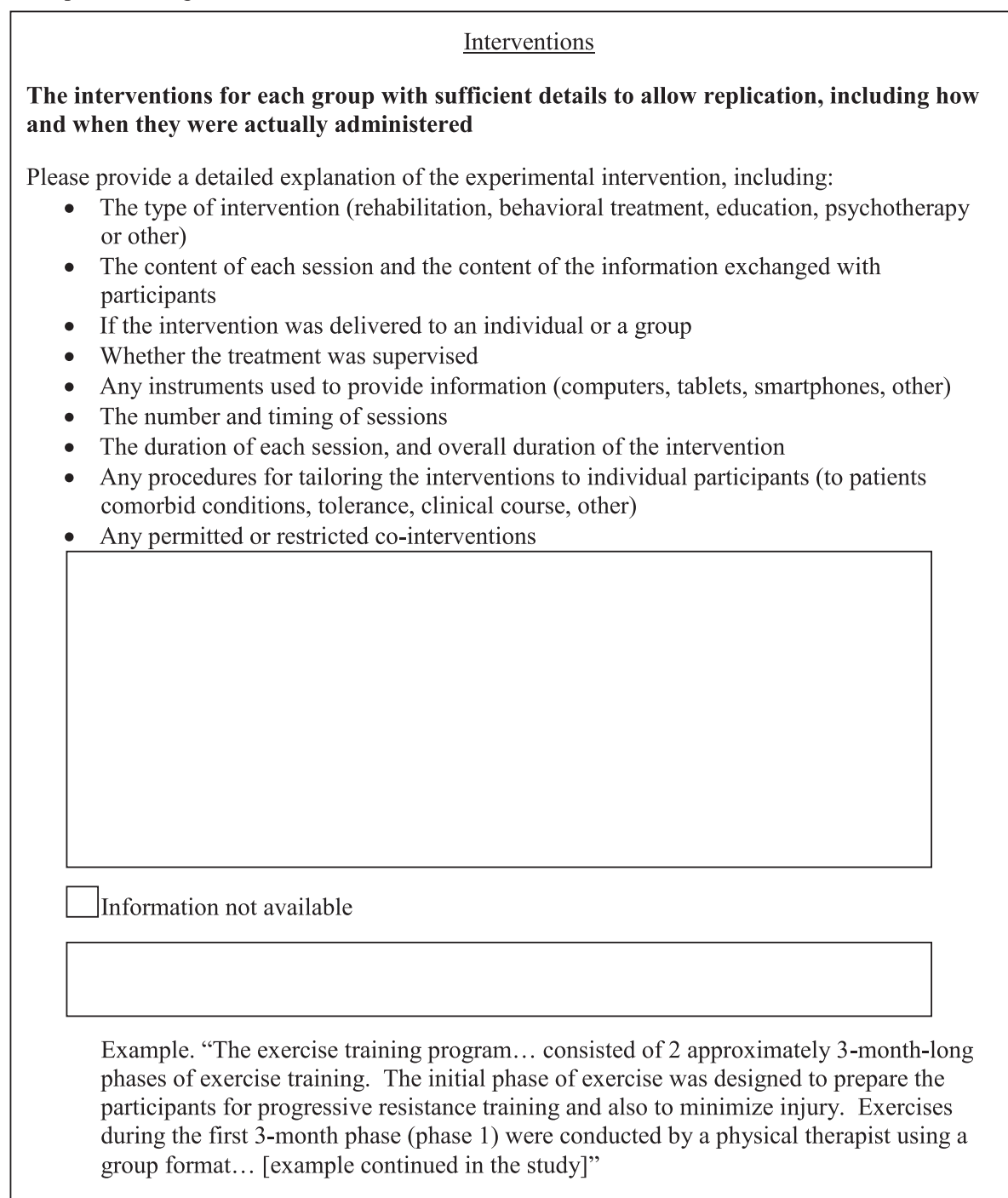

Fig. 2 Example of the experimental writing tool

stratification on the study site, the following key words were pre-specified 'Computer generated,' 'blocks of 4,' ' $1: 1$ ', 'stratification on site'. We also pre-specified a weight for each keyword.

For each protocol, completeness of reporting was determined by the presence or absence of the pre-specified keywords and their respective pre-specified weights. If the information was not available in the protocol but was described by the writer as missing, it was rated as completely reported.

Because the number of keywords varied among the domains by the domain type, the type of treatment evaluated, and the context of the protocol, we standardized the scores for each domain on a scale of $0-10$. An example for the scoring system for completeness of reporting is in Additional file 3. Therefore, we obtained six scores for completeness of reporting for each participant-protocol pair, three associated with domains written with the writing tool and three with domains written without the tool. These scores were the unit of analysis (cf. Statistical methods section), and the statistical analysis allowed for estimating mean scores for completeness of reporting with and without the writing tool.

Two independent researchers blinded to intervention assignment and to the writer identity assessed the presence of these keywords for all protocols by domain. To maintain blinding, outcome assessors measured the outcome for each of the six domains separately, all text 
appearing in a different random order in the same format (with the same font and text size). After the researchers had assessed all the domains, they met to resolve any disagreements by consensus.

\section{Secondary outcomes}

Secondary outcomes were (1) the scores for completeness of reporting for each individual domain (trial design, randomization, blinding, participants, interventions, and outcomes of participant reports) and (2) the mean score for completeness of reporting of pre-specified essential elements of each domain (Additional file 4).

\section{Ancillary study}

We aimed to compare the mean global score for completeness of reporting scores (scale 0-10) for all domains of the manuscript written by the participants with and without the tool to the methods sections of the published articles. For this purpose, we retrieved all the published reports corresponding to the selected protocols and their related appendices. The same two outcome assessors were asked to read the methods section of the published articles as well as all appendices referenced in the article and evaluate the presence or absence of the same pre-determined keywords. They were not blinded to the journal or authors' names.

\section{Sample size calculation}

The sample size was calculated using the same method as for a cluster randomized cross-over trial [29]. We assumed a mean score of 4 (0-10 scale) for the domains written without the tool (i.e. control group) and considered a standard deviation (SD) of 4 (which is a rather conservative assumption). Our hypothesis was that the mean score would be 6 for the domains written with the tool (i.e. experimental group). We specified an intraclass correlation coefficient of 0.8 (i.e. the correlation between scores of reports of two domains with the same interventional assignment, written by the same student). Such a conservative value was motivated by the nature of the design: two domains within a cluster are actually two domains completed by the same participant. We hypothesized that the intraclass correlation coefficient was half the intraclass correlation (0.4), and we considered a twosided $5 \%$ Type I error and a nominal power of $90 \%$. The inflation factor then was 1.4, the required number of observations (i.e. domains) per group 120, and the required number of participants 40 .

\section{Statistical methods}

Descriptive statistics were reported as number and percentage for categorical variables and median and interquartile range (IQR) for quantitative variables. The statistical unit of analysis was the section, which was embedded in the couple participant-protocol (since each participant had a different protocol, there is no distinction between participants and protocols). Therefore, we had six observations for each participant: three in the experimental group in which the writing aid tool was used, and three in the control group. For the main analysis, sections were considered exchangeable (i.e. the intervention effect was assumed to be the same whatever the domain of the section). Then, such a data-structure is the same as the classical data structure encountered in split-mouth designs or cluster randomized cross-over trials. Therefore, data were analyzed using a mixed model, which included a fixed intervention effect, a random participant effect, and a random participant-group effect [30].

Furthermore, because the hypothesis of a common intervention effect to all six domains was strong, we completed the primary analysis with a series of six independent substudies (i.e. one for each domain). For each of these substudies, we had only one statistical unit associated with each couple participant-protocol, which implies independence between the statistical units. Therefore, we performed classical Student $t$-tests. To evaluate the robustness of our results, we performed a sensitivity analysis with simulations of different possible weighting systems (Additional file 5).

For the ancillary study, we also considered the domain's score for completeness of reporting as the unit of analysis. For each domain, within each protocol, we had one score for the participant of the present study and another for the authors of the published report. These paired data were split by whether the participant used the writing tool or not. Differences in paired scores were then analyzed in the framework of mixed models with no other fixed effect than an intercept and with the protocol as a random effect.

\section{Results \\ Participants}

Forty-one masters and doctoral students participated in this study in May 2014. The flow diagram is shown in Fig. 3. As shown in Table 1, participants had a median age of 29 (IQR, 26-33) years. Seven participants (17\%) reported having had experience with writing about RCTs. Almost all participants $(\mathrm{n}=38(93 \%))$ reported having previously been taught about RCTs and 24 (58 \%) reported being familiar with reporting guidelines.

\section{Protocols}

We identified 158 of 308 citations screened, from which we sampled 41 protocols (one for each participant). A flowchart of the method for selecting protocols is shown in Additional file 1, and Table 1 describes the characteristics of protocols. Overall, 20 protocols reported an RCT 


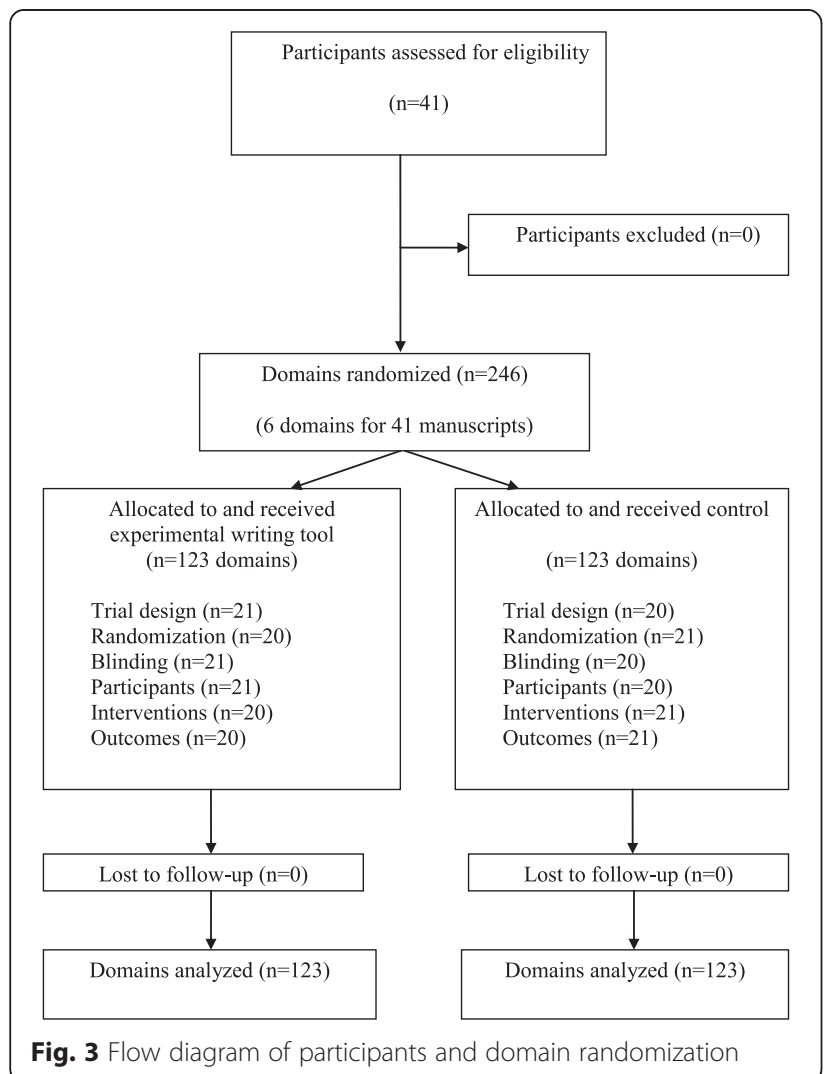

evaluating pharmacologic treatments (oral drugs, intravenous/parenteral treatments, intramuscular treatments, subcutaneous treatments, intradermal, treatment strategies) and 17 non-pharmacologic treatments (surgery or implantable device, participative interventions, radiation).

\section{Outcomes}

The mean global score for completeness of reporting (scale 0-10) for all domains was higher with than without the use of the writing aid tool: mean (SD) 7.1 (1.2) versus 5.0 (1.6), for a mean difference $(95 \% \mathrm{CI})$ of 2.1 (1.5-2.7; $P<0.0001$; Table 2).

Secondary outcomes are reported in Table 2. The completeness of reporting (score 0-10) was significantly higher with than without the writing aid tool for all domains except blinding and outcomes. The mean difference in scores $(95 \% \mathrm{CI})$ for each domain were for trial design, 5.4 (4.1-6.7), $P<0.01$; randomization, 3.8 (1.1-4.4), $P<0.01$; blinding, 0.7 ( -0.7 to 2.0 ), $P=0.50$; participants, 2.2 (0.8-3.6), $P<0.01$; interventions, 1.8 (0.7-2.9); $P<0.01$; and outcomes, -0.3 ( -2.0 to 1.3 ), $P=0.43$.

The completeness of reporting of essential elements was higher with than without the writing aid tool, with a mean difference (95\% CI) of 1.4 (0.5-2.3; $P=0.002)$.

On sensitivity analyses, the results were robust to the choice of the weight (Additional file 4).
Table 1 Participant and protocol characteristics

\begin{tabular}{|c|c|}
\hline Participant characteristics & $N=41(\%)$ \\
\hline Age, median (IQR) & $29(26-33)$ \\
\hline Gender (female) & $24(58.5 \%)$ \\
\hline Education (doctoral students) & $14(34.1 \%)$ \\
\hline \multicolumn{2}{|l|}{ Frequency reading $\mathrm{RCTs}$} \\
\hline More than once a year & $30(73.2 \%)$ \\
\hline Once a month & $6(14.6 \%)$ \\
\hline Once a week & $5(12.2 \%)$ \\
\hline Experience writing RCTs & 7 (17.1\%) \\
\hline Taught about RCTs & $38(92.7 \%)$ \\
\hline Previously involved in RCTs & $18(43.9 \%)$ \\
\hline Familiar with guidelines & $24(58.5 \%)$ \\
\hline Comfortable with English & $26(63.4 \%)$ \\
\hline Trial characteristics & $N=41(\%)$ \\
\hline Pharmacologic & $24(22.0 \%)$ \\
\hline Medication-based & 19 \\
\hline Oral & 7 \\
\hline Intravenous/parenteral & 3 \\
\hline Intramuscular & 2 \\
\hline Subcutaneous & 3 \\
\hline Intradermal & 1 \\
\hline Radiation & 3 \\
\hline Strategy & 4 \\
\hline Non-pharmacologic, surgical & $8(19.5 \%)$ \\
\hline Surgical procedures & 4 \\
\hline Implantable devices & 3 \\
\hline Non-pharmacologic, non-surgical & $9(58.5 \%)$ \\
\hline Care support & 5 \\
\hline Psychotherapy & 2 \\
\hline Patient education & 2 \\
\hline External device & 1 \\
\hline Physiotherapy & 1 \\
\hline
\end{tabular}

RCT Randomized controlled trial

\section{Ancillary study}

Results of the ancillary study are shown in Table 3. Overall, the global completeness of reporting scores for the sections written without the writing aid tool did not significantly differ from those of the published manuscripts, with a mean difference of $-0.48(-1.07$ to 0.11$)$. In contrast, the global score for completeness of reporting scores for the sections written with the writing aid tool were higher than those of the publications, with a mean difference of $+1.73(1.10-2.37), P<0.001$.

\section{Discussion}

We developed a writing aid tool to support the use of the CONSORT statement. We performed a proof-of- 
Table 2 Completeness of reporting domains of methods sections with and without the writing aid tool

\begin{tabular}{|c|c|c|c|c|}
\hline \multirow[t]{2}{*}{ Domain } & \multirow{2}{*}{$\begin{array}{l}\text { Writing aid tool scores }(0-10) \\
\text { Mean (SD) }\end{array}$} & \multirow{2}{*}{$\begin{array}{l}\text { No writing aid tool scores }(0-10) \\
\text { Mean (SD) }\end{array}$} & \multirow{2}{*}{$\begin{array}{l}\text { Mean difference } \\
(95 \% \mathrm{Cl})\end{array}$} & \multirow[t]{2}{*}{$P$ value } \\
\hline & & & & \\
\hline $\begin{array}{l}\text { Global score for completeness of reporting for } \\
\text { all domains (primary outcome) }\end{array}$ & $7.1(1.2)$ & $5.0(1.6)$ & $2.1(1.5-2.7)$ & $<0.001^{*}$ \\
\hline $\begin{array}{l}\text { Score for completeness of reporting for } \\
\text { essential elements }\end{array}$ & $7.8(1.6)$ & $6.4(2.3)$ & $1.4(0.5-2.3)$ & $0.002^{*}$ \\
\hline \multicolumn{5}{|c|}{ Completeness of reporting score by individual domain } \\
\hline Trial design & $8.1(2.3)$ & $2.7(1.9)$ & $5.4(4.1-6.7)$ & $<0.01^{* *}$ \\
\hline Randomization & $8.4(2.4)$ & $4.6(2.9)$ & $3.8(1.1-4.4)$ & $<0.01^{* *}$ \\
\hline Blinding & $6.9(2.0)$ & $6.2(2.3)$ & $0.7(-0.7$ to 2.0$)$ & $0.44^{* *}$ \\
\hline Participants & $6.7(2.0)$ & $4.5(2.4)$ & $2.2(0.8-3.6)$ & $<0.01^{* *}$ \\
\hline Interventions & $7.1(1.5)$ & $5.3(2.0)$ & $1.8(0.7-2.9)$ & $<0.01^{* *}$ \\
\hline Outcomes & $6.1(2.1)$ & $6.4(3.0)$ & $-0.3(-2.0$ to 1.3$)$ & $0.78^{* *}$ \\
\hline
\end{tabular}

*Mixed models

** Student $t$-test

concept RCT including masters and doctoral students to evaluate the impact of this tool. Our results revealed a large effect of the writing tool on the completeness of reporting scores, with a mean difference in scores of 2.1 on a $0-10$ scale. Completeness of reporting was significantly improved for all six domains of the methods section except for blinding and outcomes. However, for these domains, the scores without the use of the tool were relatively high and the opportunity for improvement was consequently limited. The ancillary study showed that the manuscripts written with the tool in a limited time (4 hours) had higher global score of completeness of reporting than the actual reports, which were published in high-impact-factor journals.

Our study has several strengths. First, it is a proof-ofconcept study that used a strong design, an RCT, to evaluate the impact of this tool. We performed an RCT using a split-manuscript design. Second, this study explored the impact of the writing tool using real protocols of published RCTs. Third, the protocols selected evaluated a wide variety of different interventions exploring pharmacologic- and non-pharmacologic-based treatments (surgical and non-surgical) as well as various treatment strategies (treatment timing, target outcomes, etc.). The quality of reporting of the selected protocols varied.
However, our study had some limitations. First, the participants were masters and doctoral students and the writing session lasted only 4 hours, which is not representative of typical authors and the typical context of writing a report of an RCT. However, the global score for completeness of reporting was not lower than that for the published report. In contrast, this comparison confirmed a large effect of the writing tool. Second, the writing aid tool considered only six domains of the methods section of a manuscript using the CONSORT 2010 statement and the extension for nonpharmacologic treatments. We did not explore the impact of the implementation of all items of the CONSORT statement and all its extensions on the completeness of reporting. Third, there is an important degree of relatedness between the experimental intervention (the writing aid tool) and the evaluation. However, the primary outcome we used (i.e. the completeness of reporting according to the CONSORT statement and CONSORT extension for nonpharmacologic treatments) is the outcome usually used to evaluate the adherence to reporting guidelines and seemed the most relevant outcome. Finally, we lack a validated outcome measure to appropriately evaluate the quality of reporting in various contexts. However, the sensitivity analysis exploring the impact of a variation of the weight used showed that our results were robust.

Table 3 Ancillary study, mean global score for completeness of reporting (scale 0-10) for all domains written with or without the writing aid tool compared to the published report

\begin{tabular}{lllll}
\hline & Manuscript written by participants & Published report & Mean difference (95 \% Cl) in global score & $P$ value $^{*}$ \\
& Mean (SD) global score & Mean (SD) global score & $\mathrm{N}=123$ domains & \\
& $\mathrm{N}=123$ domains & $\mathrm{N}=123$ domains & & 0.11 \\
\hline No writing aid tool & $5.02(2.73)$ & $5.50(2.56)$ & $-0.48(-1.07$ to 0.11$)$ & $<0.001$ \\
\hline
\end{tabular}

*Mixed models 
Previous studies have explored the impact of other types of interventions on compliance with the CONSORT guidelines when reporting RCTs. These studies have explored the impact of the publication of the main CONSORT guidelines and the CONSORT extensions studying reporting before and after [10] and over time (using a time series analysis) [20], journal endorsement of CONSORT, and implementation by a journal (i.e. requiring writers to submit a completed checklist indicating where the information is reported in the manuscript) [18, 31]. Hopewell et al. [20] showed that an active policy to enforce the CONSORT guidelines for abstracts led to an immediate increase in the completeness of reporting. Finally, the results of the ancillary study need to be interpreted with caution. In fact, we did not evaluate the readability of each section. Some sections written with the tool could be more complete but also more difficult to read. Furthermore, outcome assessors were not blinded.

In this study, we proposed a new approach to improve the reporting of RCT results and assist with the successful implementation of the CONSORT statement. In fact, most of the initiatives previously proposed to improve implementation occur after the manuscript has been written. With this writing aid tool, we aimed (1) to guide writers to use the CONSORT guidelines and (2) to oblige writers to address each item of the CONSORT guidelines while writing the first draft of their manuscript. This study has important implications for authors, editors, and researchers. It demonstrates that a writing aid tool can improve the completeness of reporting and that we need to rethink the strategies used to implement CONSORT. The tool needs to be expanded to include all CONSORT items and incorporate all extensions. The tool is freely available on the web [28] and in Additional file 2.

\section{Conclusions}

This study demonstrates that the use of a writing aid tool improves the completeness of reporting the results of RCTs. Our results indicate new opportunities to improve the quality of reporting research.

\section{Additional files}

Additional file 1: Selection of protocols. (DOCX $278 \mathrm{~kb}$ )

Additional file 2: The CONSORT-based writing tool. (DOCX $144 \mathrm{~kb}$ )

Additional file 3: The CONSORT-based writing tool. (DOCX $19 \mathrm{~kb}$ )

Additional file 4: Weights for the scoring system for the completeness of reporting essential items according to intervention type. (DOCX $18 \mathrm{~kb}$ )

Additional file 5: Sensitivity analyses. (DOCX 257 kb)

\section{Abbreviations}

IQR: Interquartile range; RCTs: Randomized controlled trials.

\section{Competing interests}

Philippe Ravaud is the director of the French EQUATOR (Enhancing the QUAlity and Transparency Of health Research) Centre and member of the EQUATOR Network Steering Group. Isabelle Boutron is the deputy director of the French EQUATOR Centre and academic editor for BMC Medical Research Methods, PLOS Medicine, PLOS ONE, and senior editor for Trials. Douglas Altman is chair of the EQUATOR Network Steering Group, director of the UK EQUATOR Centre and one of the Editors-in-Chief for Trials. This study was performed within the CONSORT (Consolidated Standards of Reporting Trials) Partnership. The other authors declare that they have no competing interests.

\section{Authors' contributions}

All authors participated in the study conception, design and drafting. BG and RP performed the statistical analysis. All authors read and approved the final manuscript.

\section{Acknowledgements}

We thank Elise Diard for participating in developing the tool. We thank Laura Smales for proofreading this manuscript.

\section{Author details}

${ }^{1}$ Centre d'Épidémiologie Clinique, Hôpital Hôtel Dieu, Assistance Publique des Hôpitaux de Paris, Hôpital Hôtel Dieu, Aile A2 1er étage 1, Place du parvis Notre Dame, 75181 Paris, Cedex 4, France. ${ }^{2}$ Paris Descartes University, Paris, France. ${ }^{3}$ INSERM, UMR 1153, Centre of Research in Epidemiology and Statistics Sorbonne Paris Cité - (CRESS), METHODS team, Paris, France. ${ }^{4}$ INSERM CIC 1415, Université François-Rabelais de Tours; CHRU de Tours, Tours, France. ${ }^{5}$ Centre for Statistics in Medicine, Nuffield Department of Orthopaedics, Rheumatology \& Musculoskeletal Sciences, University of Oxford, Oxford, UK. ${ }^{6}$ Department of Epidemiology, Columbia University Mailman School of Public Health, New York, NY, USA.

Received: 15 May 2015 Accepted: 21 August 2015

Published online: 15 September 2015

\section{References}

1. Glasziou P, Altman DG, Bossuyt P, Boutron I, Clarke M, Julious S, et al. Reducing waste from incomplete or unusable reports of biomedical research. Lancet. 2014;383:267-76.

2. Chalmers I, Glasziou P. Avoidable waste in the production and reporting of research evidence. Lancet. 2009:374:86-9.

3. Moher D, Hopewell S, Schulz KF, Montori V, Gotzsche PC, Devereaux PJ, et al. CONSORT 2010 Explanation and Elaboration: updated guidelines for reporting parallel group randomised trials. BMJ. 2010;340:c869.

4. Campbell MK, Piaggio G, Elbourne DR, Altman DG. Consort 2010 statement: extension to cluster randomised trials. BMJ. 2012;345:e5661.

5. Piaggio G, Elbourne DR, Pocock SJ, Evans SJ, Altman DG. Reporting of noninferiority and equivalence randomized trials: extension of the CONSORT 2010 statement. JAMA. 2012;308:2594-604

6. loannidis JP, Evans SJ, Gotzsche PC, O'Neill RT, Altman DG, Schulz K, et al. Better reporting of harms in randomized trials: an extension of the CONSORT statement. Ann Intern Med. 2004;141:781-8.

7. Boutron I, Moher D, Altman DG, Schulz KF, Ravaud P. Extending the CONSORT statement to randomized trials of nonpharmacologic treatment: explanation and elaboration. Ann Intern Med. 2008;148:295-309.

8. CONSORT Endorsers Journals and Organizations. http://www.consort-statement. org/about-consort/endorsers. (Accessed 31/8/2015).

9. Hopewell S, Altman DG, Moher D, Schulz KF. Endorsement of the CONSORT Statement by high impact factor medical journals: a survey of journal editors and journal 'Instructions to Authors'. Trials. 2008;9:20.

10. Hopewell S, Dutton S, Yu LM, Chan AW, Altman DG. The quality of reports of randomised trials in 2000 and 2006: comparative study of articles indexed in PubMed. BMJ. 2010;340:c723.

11. Glasziou P, Meats $E$, Heneghan C, Shepperd S. What is missing from descriptions of treatment in trials and reviews? BMJ. 2008;336:1472-4.

12. Schiller P, Burchardi N, Niestroj M, Kieser M. Quality of reporting of clinical non-inferiority and equivalence randomised trials - update and extension. Trials. 2012:13:214

13. Ntala C, Birmpili P, Worth A, Anderson NH, Sheikh A. The quality of reporting of randomised controlled trials in asthma: a systematic review. Prim Care Respir J. 2013;22:417-24. 
14. Sinha S, Ashby E, Jayaram R, Grocott MP. Quality of reporting in randomized trials published in high-quality surgical journals. J Am Coll Surg. 2009;209:565-71. e561.

15. Clark L, Schmidt U, Tharmanathan P, Adamson J, Hewitt C, Torgerson D. Poor reporting quality of key Randomization and Allocation Concealment details is still prevalent among published RCTs in 2011: a review. J Eval Clin Pract. 2013;19:703-7.

16. To MJ, Jones J, Emara M, Jadad AR. Are reports of randomized controlled trials improving over time? A systematic review of 284 articles published in high-impact general and specialized medical journals. PLoS One. 2013;8:e84779.

17. Hopewell S, Collins GS, Boutron I, Yu LM, Cook J, Shanyinde M, et al. Impact of peer review on reports of randomised trials published in open peer review journals: retrospective before and after study. BMJ. 2014;349:94145.

18. Turner L, Shamseer L, Altman DG, Schulz KF, Moher D. Does use of the CONSORT Statement impact the completeness of reporting of randomised controlled trials published in medical journals? A Cochrane review Syst Rev. 2012;1:60.

19. Ghimire S, Kyung E, Kang W, Kim E. Assessment of adherence to the CONSORT statement for quality of reports on randomized controlled tria abstracts from four high-impact general medical journals. Trials. 2012;13:77

20. Hopewell S, Ravaud P, Baron G, Boutron I. Effect of editors' implementation of CONSORT guidelines on the reporting of abstracts in high impact medical journals: interrupted time series analysis. BMJ. 2012;344:e4178.

21. Yurdakul S, Mustafa BN, Fresko I, Seyahi E, Yazici H. Brief report: inadequate description and discussion of enrolled patient characteristics and potential inter-study site differences in reports of randomized controlled trials: a systematic survey in six rheumatology journals. Arthritis Rheumatol. 2014;66:1395-9.

22. Hewitt C, Hahn S, Torgerson DJ, Watson J, Bland JM. Adequacy and reporting of allocation concealment: review of recent trials published in four general medical journals. BMJ. 2005;330:1057-8.

23. Hoffmann TC, Erueti C, Glasziou PP. Poor description of non-pharmacological interventions: analysis of consecutive sample of randomised trials. BMJ. 2013;347:f3755

24. Altman DG, Moher D, Schulz KF. Improving the reporting of randomised trials: the CONSORT Statement and beyond. Stat Med. 2012:31:2985-97.

25. Hirst A, Altman DG. Are peer reviewers encouraged to use reporting guidelines? A survey of 116 health research journals. PLoS One. 2012;7:e35621.

26. Shamseer L, Stevens A, Skidmore B, Turner L, Altman DG, Hirst A, et al. Does journal endorsement of reporting guidelines influence the completeness of reporting of health research? A systematic review protocol Syst Rev. 2012;1:24.

27. Boutron I, Ravaud P. Improving the reporting of clinical research. Br J Surg. 2009:96:449-50

28. Writing aid tool. http://cochrane.fr/writingtool/testwebsite/. (Accessed 31/8/2015)

29. Giraudeau B, Ravaud P, Donner A. Sample size calculation for cluster randomized cross-over trials. Stat Med. 2008;27:5578-85.

30. Turner RM, White IR, Croudace T. Analysis of cluster randomized cross-over trial data: a comparison of methods. Stat Med. 2007;26:274-89.

31. Cobo E, Cortes J, Ribera JM, Cardellach F, Selva-O'Callaghan A, Kostov B, et al. Effect of using reporting guidelines during peer review on quality of final manuscripts submitted to a biomedical journal: masked randomised trial. BMJ. 2011:343:d6783.

\section{Submit your next manuscript to BioMed Central and take full advantage of:}

- Convenient online submission

- Thorough peer review

- No space constraints or color figure charges

- Immediate publication on acceptance

- Inclusion in PubMed, CAS, Scopus and Google Scholar

- Research which is freely available for redistribution 